\title{
Rosette-Forming Glioneuronal Tumor
}

National Cancer Institute

\section{Source}

National Cancer Institute. Rosette-Forming Glioneuronal Tumor. NCI Thesaurus. Code C129431.

A central nervous system neoplasm mostly occurring in the fourth ventricle region. It is characterized by the presence of neurocytes forming pseudorosettes and astrocytes which contain Rosenthal fibers. Cytologic atypia is minimal. 